\title{
Central Sarcopenia, Frailty and Comorbidity as Predictor of Surgical Outcome in Elderly Patients with Degenerative Spine Disease
}

\author{
Dong Uk Kim, Hyung Ki Park, Gyeoung Hae Lee, Jae Chil Chang, Hye Ran Park, Sukh Que Park, Sung Jin Cho \\ Department of Neurosurgery, Soonchunhyang University Seoul Hospital, Soonchunhyang University College of Medicine, Seoul, Korea
}

Objective : People are living longer and the elderly population continues to increase. The incidence of degenerative spinal diseases (DSDs) in the elderly population is quite high. Therefore, we are facing more cases of DSD and offering more surgical solutions in geriatric patients. Understanding the significance and association of frailty and central sarcopenia as risk factors for spinal surgery in elderly patients will be helpful in improving surgical outcomes. We conducted a retrospective cohort analysis of prospectively collected data to assess the impact of preoperative central sarcopenia, frailty, and comorbidity on surgical outcome in elderly patients with DSD.

Methods : We conducted a retrospective analysis of patients who underwent elective spinal surgery performed from January 1, 2019 to September 30, 2020 at our hospital. We included patients aged 65 and over who underwent surgery on the thoracic or lumbar spine and were diagnosed as DSD. Central sarcopenia was measured by the 50th percentile of psoas : L4 vertebral index (PLVI) using the cross-sectional area of the psoas muscle. We used the Korean version of the fatigue, resistance, ambulation, illnesses, and loss of weight (K-FRAIL) scale to measure frailty. Comorbidity was confirmed and scored using the Charlson Comorbidity Index (CCI). As a tool for measuring surgical outcome, we used the Clavien-Dindo (CD) classification for postoperative complications and the length of stay (LOS).

Results : This study included 85 patients ( 35 males and 50 females). The mean age was $74.05 \pm 6.47$ years. Using the K-FRAIL scale, four patients were scored as robust, 44 patients were pre-frail and 37 patients were frail. The mean PLVI was $0.61 \pm 0.19$. According to the CD classification, 50 patients were classified as grade 1, 19 as grade 2, and four as grade 4 . The mean LOS was $12.35 \pm 8.17$ days. Multivariate stepwise regression analysis showed that postoperative complication was significantly associated with surgical invasiveness and K-FRAIL scale. LOS was significantly associated with surgical invasiveness and CCI. K-FRAIL scale showed a significant correlation with CCI and PLVI.

Conclusion : The present study demonstrates that frailty, comorbidity, and surgical invasiveness are important risk factors for postoperative complications and LOS in elderly patients with DSD. Preoperative recognition of these factors may be useful for perioperative optimization, risk stratification, and patient counseling.

Key Words : Frailty · Sarcopenia $\cdot$ Comorbidity $\cdot$ Spine $\cdot$ Aged $\cdot$ Postoperative complications.

- Received : March 30, 2021 •Revised : June 4, $2021 ・$ Accepted : July 10, 2021

- Address for reprints : Hyung Ki Park

Department of Neurosurgery, Soonchunhyang University Hospital, 59 Daesagwan-ro, Yongsan-gu, Seoul 04401, Korea

Tel : +82-2-709-9268, Fax : +82-2-792-5976, E-mail : schnsphk@gmail.com, ORCID : https://orcid.org/0000-0001-5110-3286

This is an Open Access article distributed under the terms of the Creative Commons Attribution Non-Commercial License (http://creativecommons.org/licenses/by-nc/4.0) which permits unrestricted non-commercial use, distribution, and reproduction in any medium, provided the original work is properly cited. 


\section{INTRODUCTION}

People are living longer and the elderly population continues to increase worldwide. The population is expected to age rapidly in most countries, including Korea. Korea became an aging society (elderly population $\geq 7 \%$ of the total population) in 2000. In 2018, Korea became an aged society (defined as an elderly population $\geq 14 \%$ of the total population), and by 2026 Korea will be a super-aged society (elderly population $\geq 20 \%$ of the total population) ${ }^{37}$. The incidence of degenerative spinal disease (DSD) in the elderly population is quite high ${ }^{36,38)}$. Therefore, we are facing more patients with DSD and initiating surgery in increasing numbers of geriatric patients ${ }^{20}$.

The incidence of perioperative complications is higher in elderly patients than in younger patients, resulting in higher morbidity and mortality in this patient population ${ }^{19)}$. Although age is associated with an increased risk of surgical complications, this does not mean that spinal surgery cannot be safely performed due to advanced age ${ }^{36)}$. However, some surgeons are reluctant to perform surgery in elderly patients because of the high risk of perioperative complications due to the patient's old age. Thus, clarifying risk of surgery in elderly patients is an important factor in decision-making and the preoperative optimization of patients ${ }^{22}$. Assessing the risk of surgery of an individual patient can be difficult, especially in the elderly, due to discrepancies between an individual's physiological age and chronological age ${ }^{17}$.

Frailty and sarcopenia have been attracting attention as factors for evaluation of physiological age in recent studies of risk factors for surgery in elderly patients ${ }^{16,26)}$. Frailty is defined as a medical syndrome characterized by a decline in strength and endurance and reduced physiologic function that increase the vulnerability for increased dependency ${ }^{311}$. Frailty is a universal risk factor for recurrent falls, delirium, institutionalization, and poor postoperative outcome ${ }^{26)}$. Recently, there has been interest in the gerontology field in quantifying the increased risk of perioperative complications from frailty ${ }^{1,22,35,38)}$. The physical phenotype of frailty shares the characteristic of decrease of muscle strength, similar to sarcopenia ${ }^{4)}$. The term "sarcopenia" is used to indicate progressive reduced muscle mass, reduced muscle strength, and reduced function that affects older adults ${ }^{10,28)}$. Recent studies have reported that central sarcopenia, as measured by psoas cross-sectional area, has been associated with adverse events after cardiovascular sur- gery ${ }^{2,18,24,30,39)}$. However, the impact of central sarcopenia in spine surgery for elderly patients has not yet been well characterized. Understanding the significance and association of frailty and central sarcopenia as risk factors for spinal surgery in elderly patients will be helpful in improving surgical outcomes.

We conducted a retrospective cohort analysis of prospectively collected data to assess the impact of preoperative central sarcopenia, frailty, and comorbidity on surgical outcome in elderly patients with DSD.

\section{MATERIALS AND METHODS}

\section{Study design}

This study protocol was approved by the Institutional Review Board (IRB) and Ethics Committee of Soonchunhyang University Seoul Hospital (IRB number : 2020-11-030). We conducted a retrospective analysis on patients who underwent elective spinal surgery performed from January 1, 2019 to September 30, 2020 at Soonchunhyang University Seoul Hospital. We included patients aged 65 and over who underwent surgery on the thoracic or lumbar spine and who were diagnosed as DSD. We excluded cases of infection, trauma, reoperation, tumor, and infection. Patient characteristics, such as age, sex, body mass index (BMI), and body surface area (BSA), were collected using electronic medical records.

\section{Central sarcopenia}

There are two main methods for measuring sarcopenia : measuring muscle performance or imaging muscle mass ${ }^{10)}$. We measured central sarcopenia by measuring the cross-sectional area of the psoas muscle ${ }^{15}$. Using preoperative computed tomography (CT) scan, the cross-sectional areas of the left and right psoas muscles were calculated at the level of the L4 pedicle. To reduce error, these measurements was calculated by two researchers and averaged. To normalize for body habitus, the cross-sectional area of the L4 vertebral body was also recorded at this level (Fig. 1). We calculated the 50th percentile of psoas : L4 vertebral index (PLVI) using the following formula ${ }^{15)}:$ [right PCSA $\left(\mathrm{mm}^{2}\right)+$ left PCSA $\left.\left(\mathrm{mm}^{2}\right) / 2\right] /$ L4 vertebral CSA $\left(\mathrm{mm}^{2}\right)$. 


\section{Frailty}

Many studies worldwide have investigated frailty, and various methods for evaluating frailty including the frailty index have been reported ${ }^{26)}$. In this study, we used the Korean version of the fatigue, resistance, ambulation, illnesses, and loss of weight (K-FRAIL) scale to evaluate frailty. The K-FRAIL scale, based on the original FRAIL scale published by Morley et al. ${ }^{29)}$, was translated into Korean ${ }^{21)}$ and is a simplified index suitable for the Korean medical environment. The K-FRAIL scale is composed of a simple 5-item questionnaire (addressing fatigue, resistance, ambulation, illness, loss of weight) with no requisite for physical examination and is an easy screening method for frailty status. In this questionnaire, a score of 0 is considered as robust, 1 to 2 as pre-frail, and 3 to 5 as frail (Supplementary Fig. 1 and Supplementary Table 1).

\section{Comorbidity}

For the analysis of postoperative outcome, comorbidity was confirmed and scored as an evaluation index of the patient's condition before surgery. The Charlson Comorbidity Index $(\mathrm{CCI})^{32)}$ quantifies comorbidity with reference to medical records and has been used in many clinical practices as a measure of predicting patient prognosis and outcome. The CCI was calculated from the International Classification of Diseases-10 comorbidity codes, using 17 comorbidities (Supple-

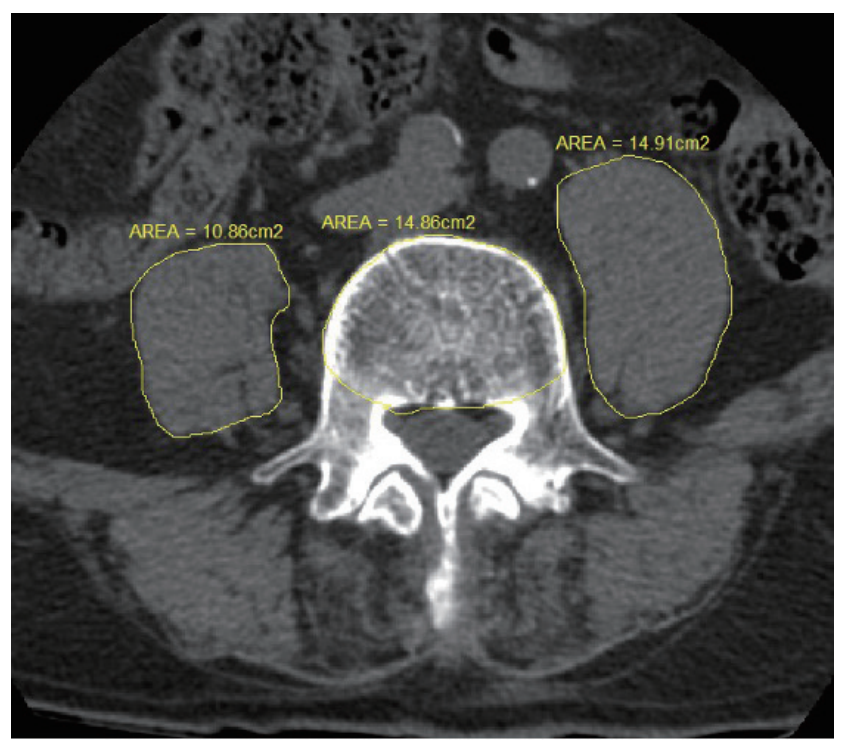

Fig. 1. The 50th percentile of psoas : L4 vertebral index (PLVI) was calculated as the ratio between the mean psoas cross-sectional area and the vertebral body cross-sectional area at the level of the $L 4$ pedicle. mentary Table 2). Each comorbidity is assigned a score (weight) when computing the weighted CCI. Diabetes with complications, hemiplegia, renal disease, and cancer are assigned a score of 2; metastatic cancer and severe liver disease are assigned a score of 3 ; human immunodeficiency virus is assigned a score of 6 ; and the remaining comorbidities are assigned a score of $1^{32)}$.

\section{Surgical invasiveness}

The type of surgery was analyzed using the surgical invasiveness index. The surgical invasiveness index is a previously validated tool that can describe the number of vertebral levels decompressed, arthrodesed, or instrumented as well as the type of approach ${ }^{9)}$. This index ranges from 0 to 48 points, and a higher the score indicates a greater surgical invasiveness. The index is the sum of six weighted surgical components : anterior decompression, anterior fusion, anterior instrumentation, posterior decompression, posterior fusion, and posterior instrumentation. The weights for each component represent the number of vertebral levels at which each is performed. The operation time was analyzed in minutes.

\section{Surgical outcome}

To measure surgical outcome, we used the presence or absence of postoperative complications and the length of stay (LOS). For postoperative complications, the Clavien-Dindo (CD) classification was used ${ }^{3)}$. This classification is graded from 1 to 5 according to increasing severity and the type of intervention required to treat the complication (Supplementary Table 3$)^{3)}$. In this study, grades II, III, IV, and V were considered as the presence of postoperative complications. LOS was calculated from the date of surgery to discharge, not the number of days from admission to discharge.

\section{Statistical analysis}

Postoperative complication and LOS were set as dependent variables, and patient variables such as sex, age, CCI, BMI, BSA, K-FRAIL scale, operation time, and surgical invasiveness index were set as independent variables. First, univariate analysis was performed; several variables were selected and multivariate linear regression analysis was performed to analyze the correlation between the variables. In addition, to investigate the correlation between the K-FRAIL scale and central sarcopenia, a correlation analysis between the K-FRAIL scale, PLVI, 
CCI was performed to calculate the significance level and correlation coefficient. All statistical analyses were performed using SPSS version 26 (IBM, Armonk, NY, USA) and statistical significance was defined as a $p$-value $<0.01$.

Table 1. Patient characteristics in the study group $(n=85)$

\begin{tabular}{lc}
\hline Variable & Value \\
\hline Age (years) & $74.05 \pm 6.47$ \\
Sex, male/female & $35(41.2) / 50(58.8)$ \\
$\mathrm{BMI}\left(\mathrm{kg} / \mathrm{m}^{2}\right)$ & $25.12 \pm 2.97$ \\
$\mathrm{BSA}\left(\mathrm{m}^{2}\right)$ & $1.64 \pm 0.19$ \\
$\mathrm{CCl}$ & \\
$\leq 2$ & $13(15.3)$ \\
3 & $23(27.0)$ \\
4 & $27(31.8)$ \\
$\geq 5$ & $22(25.9)$ \\
K-FRAlL scale & \\
Robust, 0 & $4(4.7)$ \\
Pre-frail, $1-2$ & $44(51.8)$ \\
Frail, 3-4 & $37(43.5)$ \\
PLVI & $0.61 \pm 0.19$
\end{tabular}

\section{Diagnosis}

$\begin{array}{lc}\text { Spinal stenosis } & 51(60.0) \\ \text { Herniated intervertebral disc } & 16(18.8) \\ \text { Spondylolisthesis } & 10(11.8) \\ \text { Others } & 8(9.4)\end{array}$

\begin{tabular}{|c|c|}
\hline \multicolumn{2}{|l|}{ Surgical invasiveness index } \\
\hline $1-3$ & $50(58.8)$ \\
\hline $4-7$ & $19(22.4)$ \\
\hline$\geq 8$ & $16(18.8)$ \\
\hline Operative time (minutes) & $154.84 \pm 85.54$ \\
\hline
\end{tabular}

Clavien-Dindo classification

\begin{tabular}{lc} 
Grade I & $50(58.8)$ \\
Grade II & $31(36.5)$ \\
Grade III & - \\
Grade IV & $4(4.7)$ \\
Grade V & - \\
Length of stay (days) & $12.35 \pm 8.17$ \\
\hline
\end{tabular}

Values are presented as mean \pm standard deviation or number (\%). BMI : body mass index, BSA : body surface area, $\mathrm{CCl}$ : Charlson Comorbidity Index, K-FRAIL scale: Korean version of the FRAIL, PLVI : the 50th percentile of psoas : L4 vertebral index

\section{RESULTS}

\section{Patient demographics}

A total of 128 patients were initially selected; after excluding 43 patients based on exclusion criteria as described in the MATERIALS AND METHODS, this study included 85 patients. The patient group included 35 males (41.2\%) and 50 females (58.8\%); the mean age was $74.05 \pm 6.47$ years. The average BMI and BSA were $25.12 \pm 2.97 \mathrm{~kg} / \mathrm{m}^{2}$ and $1.64 \pm 0.19 \mathrm{~m}^{2}$, respectively. Regarding the K-FRAIL scale, four patients (4.7\%) were scored as robust, 44 patients $(51.8 \%)$ were pre-frail and 37 patients (43.5\%) were frail. The mean PLVI was $0.61 \pm 0.19$. Surgical diagnoses included spinal stenosis (51 patients), herniation of intervertebral disc (16 patients), spondylolisthesis (10 patients), and other (eight patients). Regarding surgical invasiveness, 16 patients (18.8\%) were scored 8 points or more. The mean operative time was $154.84 \pm 85.54$ hours. According to the CD classification, 50 patients were classified as grade 1, 19 as grade 2 , and four as grade 4 . The mean LOS was $12.35 \pm 8.17$ days (Table 1).

\section{Postoperative complications}

Postoperative complications occurred in 35 patients (41.2\%). The most common complications were poor oral intake (16 patients, $18.8 \%$ ), voiding difficulty (14 patients, $16.5 \%)$, urinary tract infection (four patients, $4.7 \%$ ), pneumonia (three patients, 3.5\%), and delirium (three patients, 3.5\%) (Table 2). Univariate analysis showed that age, surgical invasiveness,

Table 2. Postoperative complications in patients $(n=85)$

\begin{tabular}{lc}
\hline Complication & Value \\
\hline Poor oral intake & $16(18.8)$ \\
Voiding difficulty & $14(16.5)$ \\
\hline Urinary tract infection & $4(4.7)$ \\
Delirium & $3(3.5)$ \\
Pneumonia & $3(3.5)$ \\
Postoperative hematoma & $2(2.4)$ \\
Acute kidney injury & $1(1.2)$ \\
Electrolyte imbalance & $1(1.2)$ \\
Sepsis & $1(1.2)$ \\
Cardiac arrest & $1(1.2)$ \\
Superficial wound infection & $1(1.2)$ \\
\hline
\end{tabular}

Values are presented as number (\%) 
CCI, and K-FRAIL scale were statistically significant : age (odds ratio [OR], 1.112; 95\% confidence interval [CI], 1.0331.197; $p=0.05$ ), surgical invasiveness (OR, 1.361; 95\% CI, 0.841-1.881; $p<0.001)$, CCI (OR, 1.923; 95\% CI, 1.270-2.911; $p=0.002$ ), and K-FRAIL scale (OR, 0.102; 95\% CI, 0.009$0.158 ; p=0.001)$. Multivariate stepwise regression analysis showed that postoperative complication was significantly associated with surgical invasiveness $(p<0.001)$ and K-FRAIL scale $(p=0.006)$ (Table 3$)$.

\section{LOS}

The mean LOS was $12.35 \pm 8.17$ days. Univariate analysis showed that age, operation time, surgical invasiveness, CCI, and K-FRAIL scale were statistically significant : age (OR, 0.416; 95\% CI, 0.156-0.676; $p=0.002$ ), operation time (OR, 0.026; 95\% CI, 0.06-0.046; $p=0.011$ ), surgical invasiveness (OR, 1.361; 95\% CI, 0.841-1.881; $p<0.001$ ), CCI (OR, 3.150; 95\% CI, 1.878-4.423; $p<0.001)$, and K-FRAIL scale (OR, 2.612; 95\% CI, 0.983-4.241; $p=0.002$ ). Multivariate stepwise regression analysis was performed, and LOS was associated with surgical invasiveness (OR, 1.088; 95\% CI, 0.606-1.571; $p<0.001)$ and CCI (OR, 2.204; 95\% CI, 0.974-3.435; $p=0.001)$ (Table 4).

\section{Central sarcopenia and frailty}

Pearson correlation analysis was performed between the K-

Table 3. Analysis of variables for postoperative complication

\begin{tabular}{|c|c|c|c|c|}
\hline \multirow{2}{*}{ Variable } & \multicolumn{2}{|c|}{ Univariate analysis } & \multicolumn{2}{|c|}{ Multivariate analysis } \\
\hline & OR $(95 \% \mathrm{Cl})$ & $p$-value & OR $(95 \% \mathrm{Cl})$ & $p$-value \\
\hline Sex, M/F & 0.64 (0.265 to 1.546) & 0.322 & - & - \\
\hline Age & 1.112 (1.033 to 1.197) & 0.005 & - & - \\
\hline Operation time & 1.008 (1.000 to 1.015$)$ & 0.038 & - & - \\
\hline Surgical invasiveness* & 0.082 (0.05 to 0.114$)$ & $<0.001$ & 0.068 (0.037 to 0.098$)$ & $<0.001$ \\
\hline $\mathrm{CCl}$ & 1.923 (1.270 to 2.911$)$ & 0.002 & 0.068 (-0.010 to 0.146$)$ & 0.085 \\
\hline BMI & 0.861 (0.737 to 1.007 ) & 0.061 & - & - \\
\hline BSA & 0.086 (0.007 to 1.013$)$ & 0.051 & - & - \\
\hline PLVI & 0.102 (0.009 to 0.158 ) & 0.066 & - & - \\
\hline K-FRAIL scale* & 0.102 (0.009 to 0.158 ) & $0.001^{*}$ & 0.130 (0.039 to 0.222$)$ & 0.006 \\
\hline
\end{tabular}

${ }^{*} p<0.01$. OR : odds ratio, $\mathrm{Cl}$ : confidence interval, $\mathrm{M}$ : male, $\mathrm{F}$ : female, $\mathrm{CCl}$ : Charlson Comorbidity Index, BMl : body mass index, BSA : body surface area, PLVI : the 50th percentile of psoas : L4 vertebral index, K-FRAIL scale : Korean version of the FRAIL scale

Table 4. Analysis of variables for length of stay

\begin{tabular}{|c|c|c|c|c|}
\hline \multirow{2}{*}{ Variable } & \multicolumn{2}{|c|}{ Univariate analysis } & \multicolumn{2}{|c|}{ Multivariate analysis } \\
\hline & OR $(95 \% \mathrm{Cl})$ & $p$-value & OR $(95 \% \mathrm{CI})$ & $p$-value \\
\hline Sex, M/F & $0.64(0.265$ to 1.546$)$ & 0.907 & - & - \\
\hline Age & 0.416 (0.156 to 0.676$)$ & 0.002 & - & - \\
\hline Operation time & 0.026 (0.06 to 0.046$)$ & 0.011 & - & - \\
\hline Surgical invasiveness* & 1.361 (0.841 to 1.881$)$ & $<0.001$ & 1.088 (0.606 to 1.571$)$ & $<0.001$ \\
\hline $\mathrm{CCl}^{*}$ & 3.150 (1.878 to 4.423$)$ & $<0.001$ & 2.204 (0.974 to 3.435$)$ & 0.001 \\
\hline BMI & $-0.408(-1.001$ to 0.185$)$ & 0.175 & - & - \\
\hline BSA & $-4.105(-13.693$ to 5.483$)$ & 0.397 & - & - \\
\hline PLVI & $-3.881(-13.067$ to 5.305$)$ & 0.403 & - & - \\
\hline K-FRAIL scale & 2.612 (0.983 to 4.241) & 0.002 & 1.171 (-0.278 to 2.621$)$ & 0.112 \\
\hline
\end{tabular}

${ }^{*} p<0.01$. OR : odds ratio, $\mathrm{Cl}$ : confidence interval, $\mathrm{M}$ : male, $\mathrm{F}$ : female, $\mathrm{CCl}$ : Charlson Comorbidity Index, BMl : body mass index, BSA : body surface area, PLVI : the 50th percentile of psoas : L4 vertebral index, K-FRAIL scale : Korean version of the FRAIL scale 


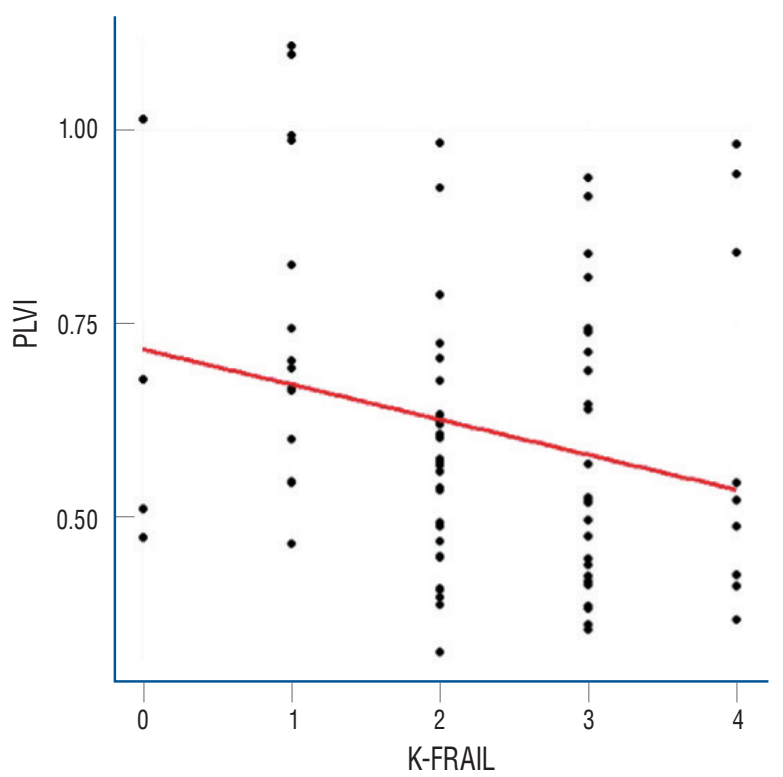

Fig. 2. Pearson correlation between K-FRAIL and PLVI (coefficient, -0.242 ; $p=0.026)$. PLVI : the 50th percentile of psoas : L4 vertebral index, K-FRAIL scale : Korean version of the FRAIL scale.

FRAIL scale and PLVI. The Pearson correlation coefficient was -0.242 , and the $p$-value was 0.026 . This showed a significant negative correlation (Fig. 2).

\section{DISCUSSION}

There is a general assumption that the outcomes of spine surgery in the geriatric population are worse than in the young and middle-aged populations. However, this has not been demonstrated in the literature ${ }^{1,38)}$. The most important reason for this may be due to widely varying discrepancies between an individual patient's physiological age and chronological $\operatorname{age}^{17)}$. Therefore, frailty and sarcopenia have been attracting attention as factors for evaluating the physiological state of elderly patients.

Frailty is characterized by physiological age-related decreases in energy, cognitive function, physical health, and other deficits that collectively make the patient more vulnerable to stressors, resulting in adverse outcomes ${ }^{4,6,17)}$. Consensus on how best to define and measure frailty remains elusive ${ }^{1,2,5}$. Frailty has been measured using different methods in the literature. Two major models of defining frailty are the frailty phenotype and the deficit accumulation model, also known as the frailty index ${ }^{26,35)}$. Segal et al. ${ }^{33)}$ found that higher scores from the 5-item modified Frailty Index were associated with an increased rate of complications and readmission in patients who underwent kyphoplasty. A modified Frailty Index based on the Canadian Study of Health and Aging-Frailty Index, which comprises 11 items, has also been proven effective in various surgical populations ${ }^{23,26)}$. However, this index does not adequately reflect physical phenotype. An ideal tool for assessing frailty would be quick and cost-effective, incorporating relevant criteria that are easily disseminated for clinical applications as well as research ${ }^{1)}$. The K-FRAIL scale is correlated with the frailty index and is a simple tool to screen for frailty in a clinical setting ${ }^{21)}$. The questionnaire can be relatively easily and quickly administered for estimating the physical phenotype. To the best of our knowledge, this is the first study using the K-FRAIL scale screening method for spine surgery of the elderly population.

Previous evidence has indicated that higher levels of frailty correlate independently with mortality, postoperative complications, prolonged hospital LOS, and discharge to a rehabilitation facility in surgery of elderly populations $\mathrm{s}^{1,17,20,22,23,26,35,38)}$. Our study showed that postoperative complications were significantly associated with frailty measured by the K-FRAIL scale. Spinal surgeons need to recognize the correlation between frailty and preoperative risk in the elderly population. The ability of a frailty index to estimate postoperative complications depends on the index used to measure frailty. Therefore, tools to assess frailty are even more crucial. The K-FRAIL scale is recommended as a screening test for the evaluation of frailty in spinal surgery for elderly patients due to its simple, convenient, and measurement of physical phenotype.

The term "sarcopenia" is used to indicate progressive low muscle mass, low muscle strength, and low function that affect older adults ${ }^{11,16,28)}$. Sarcopenia is one element of frailty syndrome that is unaffected by acute changes in clinical status or patient effort ${ }^{2}$. Reduced grip strength of sarcopenia is common criteria in physical frailty ${ }^{11}$. The cause of sarcopenia is generally thought to be multifactorial ${ }^{11,28)}$. Sarcopenia is associated with increased adverse outcomes including a loss of independence, frailty, poor quality of life, increased healthcare costs, and mortality ${ }^{11,14,28}$. Although the prevalence of sarcopenia may vary in the same cohort due to different diagnostic criteria, the prevalence of sarcopenia globally will continue to increase because of the overall increase in aging populations ${ }^{10,37)}$. Diagnosis of sarcopenia requires measurement of a 
combination of muscle mass, muscle strength, and physical performance ${ }^{10,11)}$. The most effective assessment for diagnosis involves the use of dual energy X-ray absorptiometry, bioelectrical impedance analysis, CT, and magnetic resonance imaging (MRI), and these can also have a role in some setting associated with other diseases ${ }^{11,14)}$. CT and MRI are mostly used in diagnosis and when needed for the follow-up of changed conditions in patients with DSD. Therefore, evaluation of sarcopenia using CT and MRI can be very useful for patients with spinal diseases.

Central sarcopenia through measuring psoas muscle has recently been studied as a predictor of outcome in elderly medical patients ${ }^{24)}$. Psoas muscle area (PMA) can be measured on CT scans and has been shown to correlate well with whole body muscle mass ${ }^{18,34)}$. In cardiac transplant patients, lower PMA is also associated with increased in-hospital mortality or major morbidity and worse long-term survival ${ }^{2,6,39)}$. The psoas muscle impacts functional recovery after major surgery. A validated method for quantifying sarcopenia is measurement of the PMA ${ }^{2,18,24,39)}$. PMA can be quantified using cross-sectional CT scans with good inter-observer correlation, and PMA correlates with sarcopenia ${ }^{2,24)}$. The PLVI using CT scan in our study has the advantage that it can be easily measured in all patients with spinal disease ${ }^{15}$. However, whether PMA is the most sensitive or specific indicator for frailty has not yet been determined ${ }^{2)}$.

In our study, central sarcopenia assessed using PLVI on CT was statistically associated with the K-FRAIL scale but was not strongly associated with postoperative complication and LOS. Our results suggest that PLVI is associated with frailty but is not a significant risk factor for postoperative outcomes. Whether PLVI can accurately reflect sarcopenia and whether sarcopenia is less associated with the outcome of spinal surgery should be investigated, and further research is required to address these questions.

Comorbidity is an established risk factor for complications during spine surgery ${ }^{27,38}$. In 1987, Charlson et al. ${ }^{8)}$ developed an age-based CCI score, but subsequent studies showed that age and CCI should be examined separately in multivariable predictor analyses of surgical outcomes, especially with large sample sizes ${ }^{7}$. In our study, a higher CCI appeared to associate with increased LOS after surgery. In spinal surgery of elderly patients, comorbidities should be concerned before planning surgery.
Fusion surgery leads to more complications than decompression surgery due to increased blood loss, longer operation time, and higher infection rate ${ }^{20,22)}$. Dagistan et al. ${ }^{12)}$ concluded that increased operative times and instrumentation and fusion procedures were strongly associated with an increased risk of developing complications. Kobayashi et al. ${ }^{25)}$ concluded that reducing surgical invasiveness is important in treatment of very elderly patients. Deyo et al. ${ }^{13)}$ showed that as the complexity of surgery increases, the risk of life-threatening complications also increases. The surgical invasiveness index used in our study is a previously validated instrument that accounts for the number of vertebral levels decompressed, arthrodesed, or instrumented as well as the surgical approach ${ }^{9}$. Our study demonstrated that as the surgical invasiveness increased, postoperative complications and LOS increased. These findings further validate the importance of surgical invasiveness when performing spine surgery in elderly patients.

Historically, surgeons have relied on clinical experience to determine patients for surgery, but risk assessment and stratification also ascertain the ability of patients to tolerate surgery. Accurate and timely risk stratification before surgery is crucial to ensure successful outcomes, especially among older and higher risk populations ${ }^{1,38)}$. A risk assessment must be made for each patient in a specific surgery. This requires a systematic and integrated approach and evaluation of risk factors related to surgery and patients. In our study, we examined the relationship of complications by CD classification using the K-FRAIL scale, PMA measurements and the surgical invasiveness index, which can be conveniently used in the outpatient clinic. The findings of our study have important implications in risk stratification, perioperative planning, and the counseling of elderly patients with DSD. Assessment of risk factors should be implemented in the pre-surgical period, including during pre-rehabilitation, nutritional supplementation, medication adjustment, and preoperative medication ${ }^{38)}$.

\section{Limitations}

This study has several limitations. First, there were only a small number of patients in the study. Second, there is a bias associated with data collection through the national insurance system. In our study, the result for no correlation between the LOS and the K-FRAIL scale is highly likely to have been distorted by an insurance system in which the LOS is limited by the type of surgery and disease. Finally, we did not assess the 
association between PLVI using CT scan and sarcopenia. This fact limits the interpretation of the relationship between PVLI and surgical outcomes. This requires further research.

\section{CONCLUSION}

The present study demonstrates that frailty, comorbidity, and surgical invasiveness are important risk factors for postoperative complication and LOS in elderly patients with DSD. Preoperative recognition of these factors may be useful for perioperative optimization, risk stratification, and patient counseling. Central sarcopenia assessed by PLVI using CT scan was associated with the K-FRAIL scale. Future research should further assess the significance of central sarcopenia in this scale as a risk factor for surgical outcome.

\section{CONFLICTS OF INTEREST}

Sukh Que Park has been editorial board of JKNS since November 2018. He was not involved in the review process of this original article. No potential conflict of interest relevant to this article was reported.

\section{INFORMED CONSENT}

This type of study does not require informed consent.

\section{AUTHOR CONTRIBUTIONS}

\author{
Conceptualization : HKP \\ Data curation : DUK, GHL \\ Formal analysis : DUK, HKP, GHL \\ Funding acquisition : JCC \\ Methodology : HRP \\ Project administration : SJC \\ Visualization: SQP \\ Writing - original draft : DUK \\ Writing - review \& editing : HKP
}

\section{ORCID}

$\begin{array}{ll}\text { Dong Uk Kim } & \text { https://orcid.org/0000-0003-0180-7642 } \\ \text { Hyung Ki Park } & \text { https://orcid.org/0000-0001-5110-3286 } \\ \text { Gyeoung Hae Lee } & \text { https://orcid.org/0000-0002-3502-469X } \\ \text { Jae Chil Chang } & \text { https://orcid.org/0000-0003-1366-1046 } \\ \text { Hye Ran Park } & \text { https://orcid.org/0000-0003-0506-4882 } \\ \text { Sukh Que Park } & \text { https://orcid.org/0000-0001-5899-179X } \\ \text { Sung Jin Cho } & \text { https://orcid.org/0000-0002-7383-591X }\end{array}$

\section{- Acknowledgements}

This work was supported by the Soonchunhyang University Research Fund.

\section{- Supplementary materials}

The online-only data supplement is available with this article at https://doi.org/10.3340/jkns.2021.0074.

\section{References}

1. Agarwal N, Goldschmidt E, Taylor T, Roy S, Dunn SCA, Bilderback A, et al. : Impact of frailty on outcomes following spine surgery: a prospective cohort analysis of 668 patients. Neurosurgery 88 : 552-557, 2021

2. Balsam LB : Psoas muscle area: a new standard for frailty assessment in cardiac surgery? J Thorac Dis 10(Suppl 33) : S3846-S3849, 2018

3. Camino Willhuber $G$, Elizondo C, Slullitel P : Analysis of postoperative complications in spinal surgery, hospital length of stay, and unplanned readmission: application of Dindo-Clavien classification to spine surgery. Global Spine J 9 : 279-286, 2019

4. Cesari M, Calvani R, Marzetti E : Frailty in older persons. Clin Geriatr Med 33 : 293-303, 2017

5. Chang SY, Son J, Park SM, Chang BS, Lee CK, Kim H : Predictive value of comprehensive geriatric assessment on early postoperative complications following lumbar spinal stenosis surgery: a prospective cohort study. Spine (Phila Pa 1976) 45 : 1498-1505, 2020

6. Charest-Morin R, Street J, Zhang H, Roughead T, Ailon T, Boyd M, et al. : Frailty and sarcopenia do not predict adverse events in an elderly population undergoing non-complex primary elective surgery for degenerative conditions of the lumbar spine. Spine J 18 : 245-254, 2018

7. Charlson M, Szatrowski TP, Peterson J, Gold J : Validation of a combined comorbidity index. J Clin Epidemiol 47 : 1245-1251, 1994

8. Charlson ME, Pompei P, Ales KL, MacKenzie CR : A new method of classifying prognostic comorbidity in longitudinal studies: development and validation. J Chronic Dis 40 : 373-383, 1987

9. Cizik AM, Lee MJ, Martin BI, Bransford RJ, Bellabarba C, Chapman JR, 
et al. : Using the spine surgical invasiveness index to identify risk of surgical site infection: a multivariate analysis. J Bone Joint Surg Am 94 : 335-342, 2012

10. Cruz-Jentoft AJ, Bahat $G$, Bauer J, Boirie $Y$, Bruyère 0 , Cederholm T, et al. : Sarcopenia: revised European consensus on definition and diagnosis. Age Ageing 48 : 16-31, 2019

11. Cruz-Jentoft AJ, Sayer AA : Sarcopenia. Lancet 393 : 2636-2646, 2019

12. Dagistan Y, Dagistan E, Gezici AR, Cancan SE, Bilgi M, Cakir U : Effects of minimally invasive decompression surgery on quality of life in older patients with spinal stenosis. Clin Neurol Neurosurg 139 : 86-90, 2015

13. Deyo RA, Mirza SK, Martin BI, Kreuter W, Goodman DC, Jarvik JG : Trends, major medical complications, and charges associated with surgery for lumbar spinal stenosis in older adults. JAMA 303 : 1259-1265, 2010

14. Dhillon RJ, Hasni $S$ : Pathogenesis and management of sarcopenia. Clin Geriatr Med 33 : 17-26, 2017

15. Ebbeling L, Grabo DJ, Shashaty M, Dua R, Sonnad SS, Sims CA, et al. : Psoas:lumbar vertebra index: central sarcopenia independently predicts morbidity in elderly trauma patients. Eur J Trauma Emerg Surg 40 : 57-65, 2014

16. Gibbons D, Ahern DP, Curley AE, Kepler CK, Butler JS : Impact of sarcopenia on degenerative lumbar spondylosis. Clin Spine Surg 34 : 4350, 2021

17. Hannah TC, Neifert SN, Caridi JM, Martini ML, Lamb C, Rothrock RJ, et al. : Utility of the hospital frailty risk score for predicting adverse outcomes in degenerative spine surgery cohorts. Neurosurgery 87 : 1223-1230, 2020

18. Indrakusuma R, Zijlmans JL, Jalalzadeh H, Planken RN, Balm R, Koelemay MJW : Psoas muscle area as a prognostic factor for survival in patients with an asymptomatic infrarenal abdominal aortic aneurysm: a retrospective cohort study. Eur J Vasc Endovasc Surg 55 : 83-91, 2018

19. Jain A, Hassanzadeh $H$, Puvanesarajah V, Klineberg EO, Sciubba DM, Kelly MP, et al. : Incidence of perioperative medical complications and mortality among elderly patients undergoing surgery for spinal deformity: analysis of 3519 patients. J Neurosurg Spine 27 : 534-539, 2017

20. Jang HJ, Chin DK, Park JY, Kuh SU, Kim KS, Cho YE, et al. : Influence of frailty on life expectancy in octogenarians after lumbar spine surgery. Neurospine $18:$ 303-310, 2021

21. Jung HW, Yoo HJ, Park SY, Kim SW, Choi JY, Yoon SJ, et al. : The Korean version of the FRAIL scale: clinical feasibility and validity of assessing the frailty status of Korean elderly. Korean J Intern Med 31 : 594-600, 2016

22. Kang T, Park SY, Lee JS, Lee SH, Park JH, Suh SW : Predicting postoperative complications in patients undergoing lumbar spinal fusion by using the modified five-item frailty index and nutritional status. Bone Joint J 102-B : 1717-1722, 2020

23. Kim JY, Park IS, Kang DH, Lee YS, Kim KT, Hong SJ : Prediction of risk factors after spine surgery in patients aged $>75$ years using the modified frailty index. J Korean Neurosurg Soc 63 : 827-833, 2020

24. Kleczynski P, Tokarek T, Dziewierz A, Sorysz D, Bagienski M, Rzeszutko $L$, et al. : Usefulness of psoas muscle area and volume and frailty scoring to predict outcomes after transcatheter aortic valve implantation. Am J
Cardiol 122 : 135-140, 2018

25. Kobayashi K, Imagama S, Sato K, Kato F, Kanemura T, Yoshihara H, et al. : Postoperative complications associated with spine surgery in patients older than 90 years: a multicenter retrospective study. Global Spine J $8: 887-891,2018$

26. Kweh B, Lee H, Tan T, O'Donohoe T, Mathew J, Fitzgerald M, et al. : Spinal surgery in patients aged 80 years and older: risk stratification using the modified frailty index. Global Spine J 11 : 525-532, 2021

27. Mannion AF, Bianchi G, Mariaux F, Fekete TF, Reitmeir R, Moser B, et al. : Can the Charlson Comorbidity Index be used to predict the ASA grade in patients undergoing spine surgery? Eur Spine J 29 : 2941-2952, 2020

28. Matsuo S, Kawakami M, Minetama M, Nakagawa M, Teraguchi M, Kagotani $R$, et al. : Clinical features of sarcopenia in patients with lumbar spinal stenosis. Spine (Phila Pa 1976) 45 : E1105-E1110, 2020

29. Morley JE, Malmstrom TK, Miller DK : A simple frailty questionnaire (FRAIL) predicts outcomes in middle aged African Americans. J Nutr Health Aging $16: 601-608,2012$

30. Paknikar R, Friedman J, Cron D, Deeb GM, Chetcuti S, Grossman PM, et al. : Psoas muscle size as a frailty measure for open and transcatheter aortic valve replacement. J Thorac Cardiovasc Surg 151 : 745-751, 2016

31. Rodríguez-Mañas L, Féart C, Mann G, Viña J, Chatterji S, Chodzko-Zajko W, et al. : Searching for an operational definition of frailty: a Delphi method based consensus statement: the frailty operative definitionconsensus conference project. J Gerontol A Biol Sci Med Sci 68 : 62-67, 2013

32. Roffman CE, Buchanan J, Allison GT : Charlson comorbidities index. J Physiother 62 : 171, 2016

33. Segal DN, Wilson JM, Staley C, Michael KW : The 5 -item modified frailty index is predictive of 30-day postoperative complications in patients undergoing kyphoplasty vertebral augmentation. World Neurosurg 116 : e225-e231, 2018

34. Shen W, Punyanitya M, Wang Z, Gallagher D, St-Onge MP, Albu J, et al. : Total body skeletal muscle and adipose tissue volumes: estimation from a single abdominal cross-sectional image. J Appl Physiol (1985) 97 : 2333-2338, 2004

35. Simcox T, Antoku D, Jain N, Acosta F, Hah R : Frailty syndrome and the use of frailty indices as a preoperative risk stratification tool in spine surgery: a review. Asian Spine J 13: 861-873, 2019

36. Watanabe T, Kanayama M, Takahata M, Oda I, Suda K, Abe Y, et al. : Perioperative complications of spine surgery in patients 80 years of age or older: a multicenter prospective cohort study. J Neurosurg Spine 32 : 554-558, 2020

37. Yoo Jl, Ha YC : Review of epidemiology, diagnosis, and treatment of osteosarcopenia in Korea. J Bone Metab 25 : 1-7, 2018

38. Zileli M, Dursun $E$ : How to improve outcomes of spine surgery in geriatric patients. World Neurosurg 140 : 519-526, 2020

39. Zuckerman J, Ades M, Mullie L, Trnkus A, Morin JF, Langlois Y, et al. : Psoas muscle area and length of stay in older adults undergoing cardiac operations. Ann Thorac Surg 103 : 1498-1504, 2017 